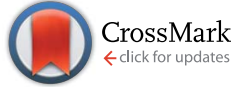

Cite this: RSC Adv., 2017, 7, 8338

Received 9th December 2016 Accepted 18th January 2017

DOI: 10.1039/c6ra27958a

www.rsc.org/advances

\title{
Preparation of 3,3,3-trifluoropropyl functionalized hydrophobic mesoporous silica and its outstanding adsorption properties for dibutyl phthalate $\uparrow$
}

\begin{abstract}
Xi Diao, ${ }^{a}$ Yun Hu, ${ }^{\star a b c}$ Yinyun He, ${ }^{a}$ Feng Quan ${ }^{a}$ and Chaohai Wei ${ }^{\text {abc }}$
In this study, 3,3,3-trifluoropropyltrimethoxysilane was first used as a modification agent to synthesize a highly hydrophobic mesoporous silica, TFP-MCM-41, using a refluxing method. The adsorption properties of TFP-MCM-41 for dibutyl phthalate (DBP) were studied by comparing its properties with that of the $n$-propyl-functionalized form of $\mathrm{MCM}-41, \mathrm{P}-\mathrm{MCM}-41$, which contains three $\mathrm{C}-\mathrm{H}$ bonds at the end of the organic chain instead of $\mathrm{C}-\mathrm{F}$ bonds as in 3,3,3-trifluoropropyltrimethoxysilane, and unmodified MCM-41. TFP-MCM-41 showed much stronger hydrophobicity, higher adsorption affinity and better selectivity for adsorption of DBP than either P-MCM-41 or MCM-41. TFP-MCM-41 could preferentially uptake DBP from an aqueous solution containing a trace of DBP and large amounts of phenol. The excellent adsorption affinity and selectivity for DBP displayed by TFP-MCM-41 were ascribed to the hydrophobic and hydrogen bond interactions between the 3,3,3-trifluoropropyl groups and DBP molecules.
\end{abstract}

\section{Introduction}

Phthalate acid esters (PAEs) are a type of plasticizer that is widely used to improve flexibility, workability and the general handling properties of certain plastics. The amount of PAEs in some plastic products can be as much as 10-60 wt\%. ${ }^{1}$ PAEs are known to be endocrine disrupting chemicals (EDCs) that may interfere with endocrine systems and pose serious risks to the health of animals and humans. ${ }^{2,3}$ Dibutyl phthalate (DBP) is a typical member of the family of PAEs and has been shown to cause deformities in aquatic organisms. ${ }^{4,5}$ As a result of its widespread use, DBP can be found throughout the environment. Due to its low water solubility and high octanol-water partition coefficient ${ }^{6}$ DBP is at present at extremely low concentrations in aqueous environments. ${ }^{7}$ However, it can pose risks even at trace levels. Furthermore, given its stability in the natural environment, DBP is bioconcentrated in living systems and biomagnified through the food chain in the ecosystem. Conventional water treatment methods; e.g., coagulation, sedimentation, filtration and disinfection, do not effectively remove DBP from treated waters. ${ }^{8}$ As a result, there is great interest in

\footnotetext{
${ }^{a}$ School of Environment and Energy, South China University of Technology, Guangzhou 510006, P. R. China. E-mail: huyun@scut.edu.cn; Tel: +86-20-39380573

${ }^{b}$ Guangdong Provincial Engineering and Technology Research Center for Environmental Risk Prevention and Emergency Disposal, Guangzhou 510006, China ${ }^{c}$ The Key Lab of Pollution Control and Ecosystem Restoration in Industry Clusters, Ministry of Education, Guangzhou 510006, P. R. China

† Electronic supplementary information (ESI) available. See DOI: 10.1039/c6ra27958a
}

the development of effective methods for removing DBP from aqueous environments. Among the treatment methods available, adsorption is a rapid and safe process that has attracted considerable attention.

The conventional adsorption materials commonly include activated carbon, ${ }^{9}$ ceramic, ${ }^{\mathbf{1 0}}$ silicon dioxide, ${ }^{\mathbf{1 1}}$ alumina, clay and mesoporous silica. ${ }^{\mathbf{1 2 - 1 4}}$ Among these materials, activated carbon and mesoporous silica are most frequently studied. Activated carbon possess very strong adsorption ability and has been used for many years in water treatment. However, it can cause an extensive adsorption for most of organic compounds in water environment, and cannot effectively separate and recycle the target pollutant, especially when the target pollutant is present at a low concentration. Hence, DBP in an aqueous solution is difficult to be enriched in activated carbon when it coexists with other pollutants with a high concentration. According to the similarity-intermiscibility theory, the hydrophobic adsorbent has a higher selectivity for DBP than other organic pollutants due to the strong hydrophobicity of DBP molecular.

Mesoporous silica materials have been widely investigated as adsorbents and as a catalytic support because of its large surface area, ${ }^{15-17}$ uniform hexagonal channels $(1.5-10 \mathrm{~nm}$ in diameter), large pore volume, and chemical stability. Unmodified mesoporous silica is highly hydrophilic due to the large number of surface silanol groups and has been used for the removal of dyes, ${ }^{18}$ nitrobenzene, ${ }^{19,20}$ and other organic pollutants. Zhao et al. ${ }^{21}$ studied the modification of MCM-41 by using the trimethylchlorosilane as the modifier, and the modified MCM-41 could still maintain the ordered mesoporous structure and possess good hydrophobicity to selectively adsorb volatile 
organic compounds. Huang et $a .^{22}$ reported that octyl and octadecyl gifted SBA-15 had a better adsorption performance for dimethyl phthalate and diethyl phthalate than unmodified SBA15 in the aqueous solution. And a higher adsorption capacity could be reached by gifting octadecyl on SBA-15. Yamashita et $a .^{23}$ studied that MCM-41 was modified with the hydrophobic triethoxyfluorosilane, attesting that organofluorine silane gifted on the mesoporous silica possess the characteristics of high thermal and chemical stability.

Adsorption properties of materials largely depend on their surface hydrophilic-hydrophobic properties, providing the basis for the interactions between adsorbents and adsorbate. ${ }^{24-27} \mathrm{~A}$ surface hydrophilicity can be easily imposed on unmodified mesoporous silica, which results in the poor adsorption affinity of hydrophobic organic compounds; therefore, surface hydrophobic modification is an effective way to enhance the adsorption capacity for organic compounds. ${ }^{28-30}$ The most common organic functional groups are alkyl groups, ${ }^{31,32}$ whose hydrophobicity increases with the increase in the length of the carbon chains. However, the presence of alkyl groups with long carbon chain occupy more channel space than alkyl groups with short carbon chain. This can lead to pore blocking, which has a negative effect on the performance of adsorbent for hydrophobic materials.

Taking these into consideration, we hope to find a new kind of hydrophobic organic modifier, which possesses a shorter carbon chain and an excellent adsorption property, simultaneously. Because of the enhanced hydrophobicity of organic functional groups with fluorine groups, in this study, 3,3,3-trifluoropropyltrimethoxysilane containing hydrophobic alkyl group and fluorine-carbon bonds was designed as a modification agent for the functionalization of MCM-41, which is a typical mesoporous silica. TFP-MCM-41 was prepared successfully by a postsynthesis grafting method. Its adsorption properties for DBP were studied in detail and in comparison with both unmodified MCM-41 and n-propyltrimethoxysilane modified MCM-41.

\section{Materials and methods}

\subsection{Materials}

All reagents used in the experiments were used without further purification. Tetraethoxysilane (TEOS, 28\% $\mathrm{SiO}_{2}$, Tianjin Fuchen Chemical Reagents Factory, China), n-cetyltrimethyl ammonium bromide (CTAB, $\geq 99.0 \%$, Bio-Green Planet) and ammonia (25-28 wt $\%$, Shanghai Richjoint Chemical Reagents Co., Ltd, China) were analytical reagent grade. 3,3,3-Trifluoropropyltrimethoxysilane (TFPTMS, 97\%, Alfa Aesar Chemical Co. Ltd., Tianjin, China), $n$ propyltrimethoxysilane (PTMS, 97\%, Sigma-Aldrich Co. LLC.) and dibutyl phthalate (DBP, $\geq 98.5 \%$, Aladdin Reagent Co. Ltd., Shanghai, China) were guaranteed reagents.

\subsection{Synthesis}

Fig. 1 is the schematic illustration of the adsorbent preparation. First, the purely siliceous MCM-41 was prepared by a hydrothermal method. ${ }^{33}$ CTAB was dissolved in the aqueous $\mathrm{NH}_{3}$ solution, and then TEOS was added dropwise with magnetic stirring at $40{ }^{\circ} \mathrm{C}$. The mole ratio in the gel mixture was
Si : CTAB : $\mathrm{NH}_{3}: \mathrm{H}_{2} \mathrm{O}=1: 0.12: 8.6: 82$. After $3 \mathrm{~h}$, the gel was transferred to an autoclave and maintained at $120{ }^{\circ} \mathrm{C}$ for $48 \mathrm{~h}$. After filtering, washing, drying and calcination at $550^{\circ} \mathrm{C}$ for $8 \mathrm{~h}$, MCM-41 was obtained.

The MCM-41 was modified by organosilane agent via a reflux method in which $0.5 \mathrm{~g}$ of MCM-41 powder was refluxed in $50 \mathrm{~mL}$ toluene with 3,3,3-trifluoropropyltrimethoxysilane (TFPTMS) or $n$ propyltrimethoxysilane (PTMS) for $18 \mathrm{~h}$ at $110{ }^{\circ} \mathrm{C}$, in which the mole ratio of the TFPTMS to MCM-41 was $0.2,0.4,0.6$ and 0.8 . Prior to the silylation, MCM-41 was dried at $100{ }^{\circ} \mathrm{C}$ for $10 \mathrm{~h}$. After the silylation, the samples were filtered and washed with adequate isopropanol and then dried at $100{ }^{\circ} \mathrm{C}$ for $10 \mathrm{~h}$. The obtained samples modified by 3,3,3-trifluoropropyltrimethoxysilane and $n$ propyltrimethoxysilane were denoted as TFP-MCM-41 and PMCM-41, respectively.

\subsection{Characterization}

$\mathrm{X}$-ray diffraction (XRD) patterns were obtained using $\mathrm{Cu} \mathrm{K} \alpha$ radiation $(\lambda=0.15418 \mathrm{~nm})$ on a Bruker D8 ADVANCE diffractometer at $40 \mathrm{kV}$ and $40 \mathrm{~mA}$ between $1^{\circ}$ and $10^{\circ}(2 \theta)$ with a step length of $0.02^{\circ}$ and a scanning rate of $0.4 \mathrm{~s}$ per step. $\mathrm{N}_{2}$ adsorption/desorption measurements were performed with a specific surface area analyzer (ASAP2020, Micrometics) at $-196{ }^{\circ} \mathrm{C}$. Prior to the measurements, samples were outgassed at $80{ }^{\circ} \mathrm{C}$ under vacuum for $4 \mathrm{~h}$. The surface areas of MCM-41, PMCM-41 and TFP-MCM-41 were determined by BET method. Fourier transform infrared (FT-IR) spectra were obtained using a Nicolet-6700 Thermo spectrometer at a spectral resolution of $1.92 \mathrm{~cm}^{-1}$. Elemental analysis was performed on an elemental analyzer (Vario EL cube) to estimate the number of organic carbon chains grafted on MCM-41 samples. Water contact angles were measured by a contact angle analyzer (OCA 20). The sample powder was uniformly dispersed on glass slide using isopropanol as a solvent, and then dried at $100{ }^{\circ} \mathrm{C}$ for $30 \mathrm{~min}$.

\subsection{Adsorption experiments and analysis methods}

$5 \mathrm{mg}$ of adsorbent was added to a conical flask containing a specific concentration of DBP in aqueous solution. The flasks were sealed and mechanically shaking in a temperaturecontrolled water bath shaker for a certain period of time at a mixing speed of $170 \mathrm{rpm}$. After the adsorption process, the solution was filtered by $0.45 \mu \mathrm{m}$ filter membrane, and the amount of the DBP adsorbed on the adsorbent was determined by the residual concentration in the filtrate by highperformance liquid chromatography (HPLC, Shimadzu LC 20AT). An HC-C18 $(250 \times 4.6 \mathrm{~mm}, 5 \mu \mathrm{m})$, (Thermo Scientific) column was used. The mobile phase was a mixture of methanol and water $(90: 10, \mathrm{v} / \mathrm{v})$ with a flow rate of $1.0 \mathrm{~mL} \mathrm{~min}^{-1}$. The UV detector was operated at $228 \mathrm{~nm}$.

The adsorption amount $(Q)$ of DBP was calculated according to the following equation:

$$
Q=\frac{V\left(C_{0}-C\right)}{m}
$$

where $Q\left(\mathrm{mg} \mathrm{g}^{-1}\right)$ is the adsorption amount of DBP, $V(\mathrm{~L})$ is the volume of the DBP solution, $C_{0}\left(\mathrm{mg} \mathrm{L}^{-1}\right)$ is the initial 

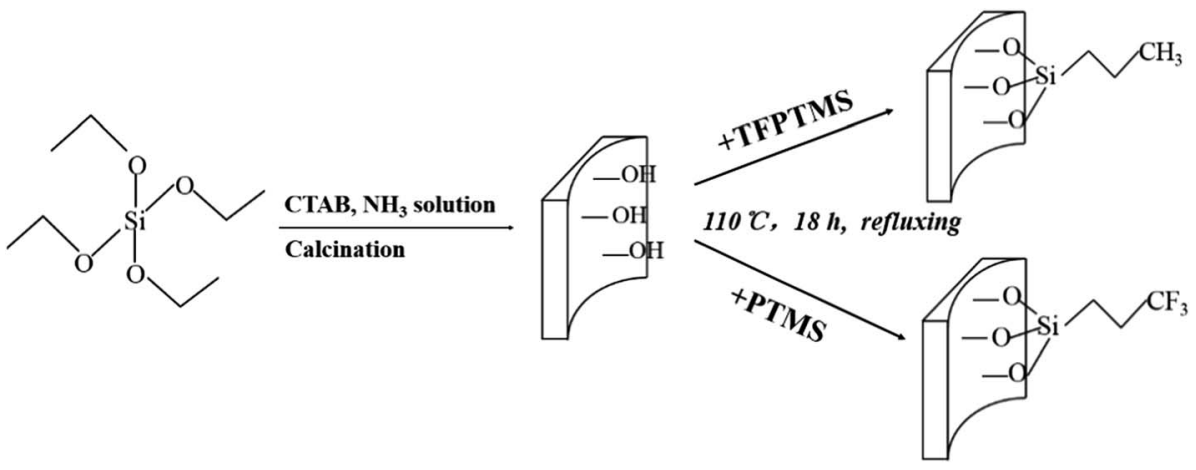

Fig. 1 The schematic illustration of the adsorbent preparation.

concentration of DBP, $C\left(\mathrm{mg} \mathrm{L}^{-1}\right)$ is the concentration of DBP in any sample time, and $m(\mathrm{~g})$ is the mass of the adsorbent used in the experiments.

For the selective adsorption experiments, a series of solutions of DBP and phenol $(\mathrm{Ph})$ in water were prepared, in which the DBP concentration was fixed at $1.8 \times 10^{-5} \mathrm{~mol} \mathrm{~L}^{-1}$ (i.e. $5 \mathrm{mg}$ $\mathrm{L}^{-1}$ ) and the molar concentrations of $\mathrm{Ph}$ were adjusted to 1,5 , 20, 50 and 100 times that of DBP. $5 \mathrm{mg}$ of adsorbent samples were suspended in $100 \mathrm{~mL}$ of the mixtures with different concentration ratios of $\mathrm{DBP}$ and $\mathrm{Ph}\left(C_{\mathrm{DBP}}: C_{\mathrm{Ph}}\right)$. The suspension solution was maintained at $25{ }^{\circ} \mathrm{C}$ and shaken continuously. After reaching adsorption equilibrium, the residual concentrations of DBP and Ph were determined by HPLC. The selective adsorption coefficient for DBP $\left(K_{\mathrm{DBP}}\right)$ was defined as the ratio of equilibrium distribution coefficient for DBP and $\mathrm{Ph}^{34}$ and was calculated via the following equation:

$$
K_{\mathrm{DBP}}=\chi_{\mathrm{D}}^{\mathrm{s}} \chi_{\mathrm{P}}^{\mathrm{a}} \chi_{\mathrm{P}}^{\mathrm{s}} \chi_{\mathrm{D}}^{\mathrm{a}}
$$

where $\chi_{\mathrm{D}}^{\mathrm{s}}$ and $\chi_{\mathrm{D}}^{\mathrm{a}}$ are the mole fractions of DBP in the aqueous solution and adsorbed to the adsorbent, $\chi_{\mathrm{P}}^{\mathrm{s}}$ and $\chi_{\mathrm{P}}^{\mathrm{a}}$ are the mole fractions of $\mathrm{Ph}$ in the aqueous solution and adsorbed to the adsorbent, respectively. If $K_{\mathrm{DBP}}>1$, the adsorbent shows adsorption selectivity for DBP, and the higher the $K_{\mathrm{DBP}}$, the stronger selectivity.

\section{Results and discussion}

\subsection{The optimization of synthetic conditions}

The FT-IR spectra of MCM-41, P-MCM-41 and TFP-MCM-41 with different TFPTMS/MCM-41 mole ratios varied from 0.2 to 0.8 are shown in Fig. 2. The peaks at 1080 and $800 \mathrm{~cm}^{-1}$ are assigned to the stretching vibrations of the $\mathrm{Si}-\mathrm{O}-\mathrm{Si}$ framework. ${ }^{15,35}$ The peaks at 2923 and $2852 \mathrm{~cm}^{-1}$, which only appear in the spectra of P-MCM-41 and TFP-MCM-41, are ascribed to the stretching vibration peak of $\mathrm{C}-\mathrm{H} .{ }^{36}$ The peaks at 1450 and $1380 \mathrm{~cm}^{-1}$ are ascribed to the anti-symmetric and symmetric bending vibrations of $\mathrm{C}-\mathrm{H}$, respectively. The peak at $1317 \mathrm{~cm}^{-1}$ is the stretching vibration of the $\mathrm{C}-\mathrm{F}$ bond..$^{37}$ These adsorption bands indicate that $n$-propyl groups and 3,3,3-trifluoropropyl groups had been successfully grafted onto the surface of MCM41. In addition, the peak at $3450 \mathrm{~cm}^{-1}$ is the stretching

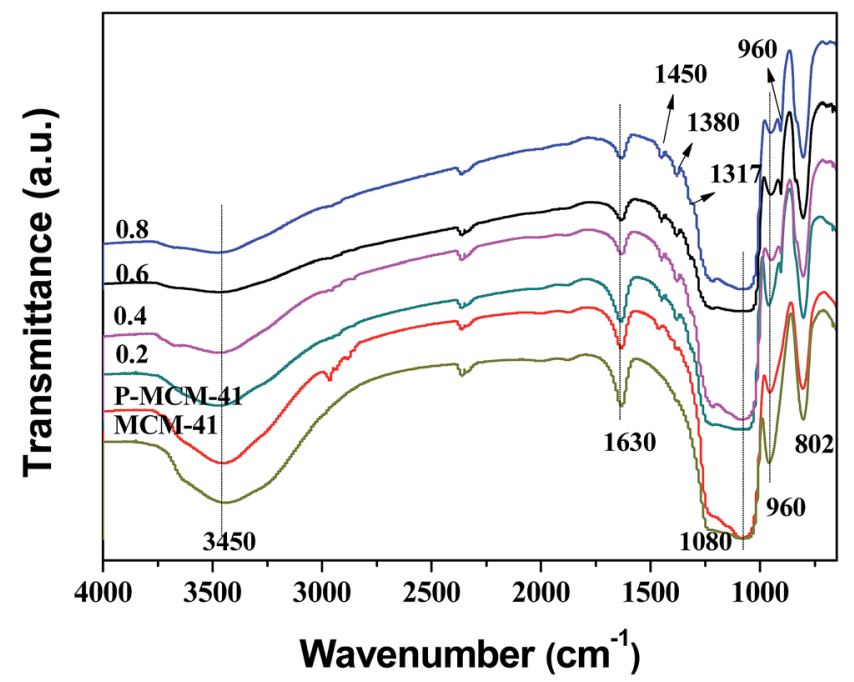

Fig. 2 FT-IR spectra of MCM-41, P-MCM-41 and TFP-MCM-41 adsorbents prepared with different modification reagent doses.

vibration peak of surface $\mathrm{O}-\mathrm{H}$ groups and adsorbed water molecules, while the peak at $1630 \mathrm{~cm}^{-1}$ is the bending vibration of the $\mathrm{H}-\mathrm{OH}$ bond. ${ }^{38}$ By comparison, the two peaks of P-MCM41 were weaker than that of MCM-41 but stronger than that in TFP-MCM-41, indicating that P-MCM-41 was more hydrophobic than MCM-41, while TFP-MCM-41 was more hydrophobic than P-MCM-41. Besides, it was found that the two peaks representing $\mathrm{O}-\mathrm{H}$ and $\mathrm{H}-\mathrm{OH}$ groups become weaker with the increase of TFPTMS/MCM- 41 mole ratio, which indicates that the materials were becoming more hydrophobic.

Fig. 3 shows the adsorption effect of the TFP-MCM-41 adsorbents prepared with different dosages of the modification reagent. The adsorption capacity increase from 38 to $65 \mathrm{mg} \mathrm{g}^{-1}$ when TFPTMS/MCM-41 mole ratio increase from 0.2 to 0.4 . However, there is only a slight increase in adsorption capacity when the TFPTMS dosage is increased further, probably indicating that the surface of the MCM-41 is nearly fully covered with TFPTMS at a TFPTMS/MCM-41 mole ratio of 0.4 . Based on the adsorption results, we determine that the optimal TFPTMS/MCM-41 is 0.4 with a theoretical TFPTMS dosage of $3.3 \mathrm{mmol} \mathrm{g}^{-1}$. 


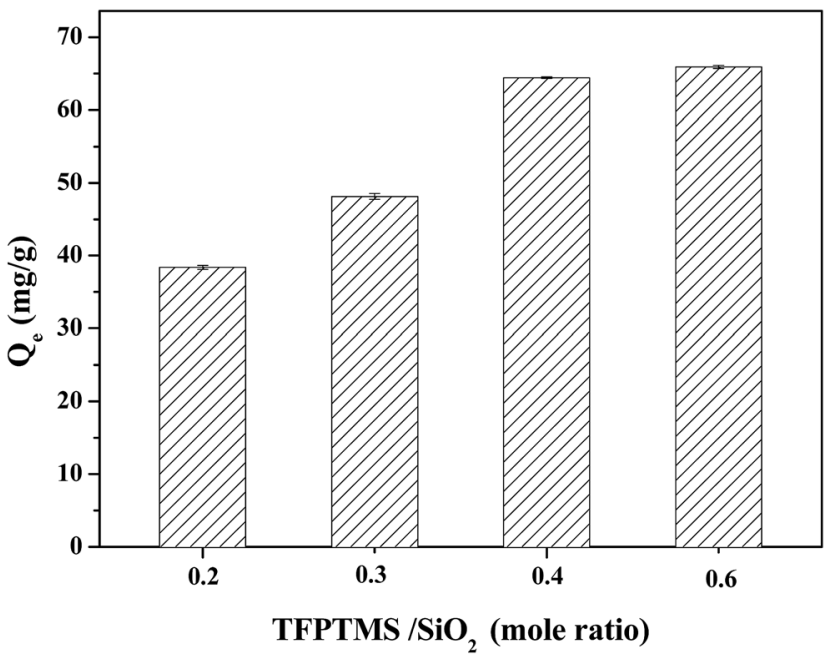

Fig. 3 Adsorption effect of TFP-MCM-41 adsorbents prepared with different doses of modification reagents.

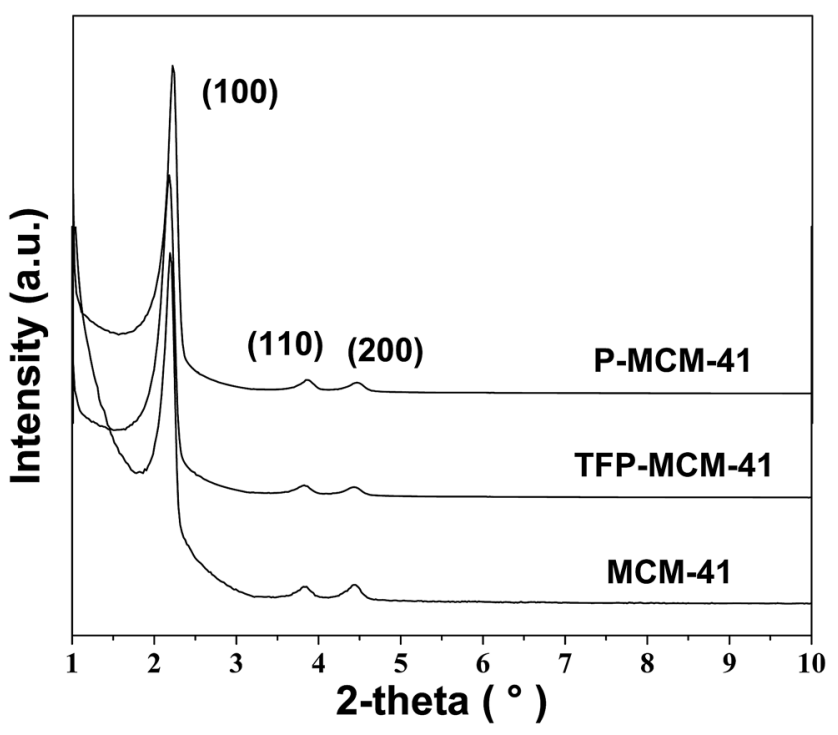

Fig. 4 XRD patterns of three unmodified and modified MCM-41 based adsorbents.

In addition, the optimal reflux temperature and time is also determined to be $110{ }^{\circ} \mathrm{C}$ and $18 \mathrm{~h}$, (Fig. S1-S6†).

\subsection{Characterization of the adsorbents}

Fig. 4 shows the XRD patterns of MCM-41, P-MCM-41 and TFPMCM-41. All samples show one strong peak near $2^{\circ}$, and two weak peaks at $3.83^{\circ}$ and $4.43^{\circ}$, corresponding to the (100), (110) and (200) diffraction planes, respectively. These data indicate that all three samples possessed typical hexagonal mesoporous structure $^{25}$ and that the long-range order of the MCM-41 solid was maintained after the grafting of the organic functional groups.

The $\mathrm{N}_{2}$ adsorption/desorption isotherms of the three samples (Fig. 5) show that they all exhibit typical type IV

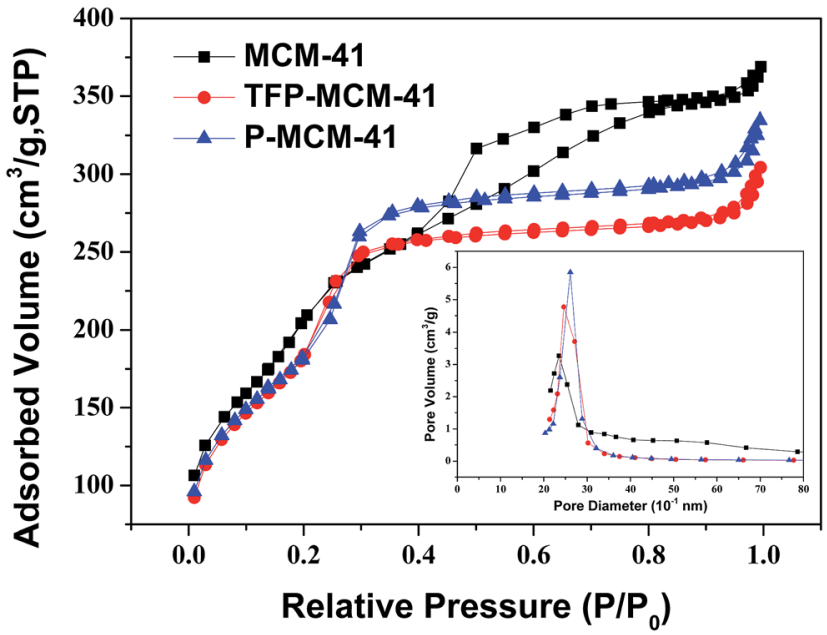

Fig. $5 \quad \mathrm{~N}_{2}$ adsorption/desorption isotherms and the pore size distribution (inset) of three unmodified and modified MCM-41 based adsorbents.

isotherms, indicative of typical materials containing mesopores. ${ }^{33}$ Table 1 contains the textural parameters of the three samples obtained from XRD and $\mathrm{N}_{2}$ adsorption/desorption data. The BET surface area, pore volume and pore diameter of MCM-41 decrease after grafting, which could be a consequence of the presence of organic surface groups. However, the open channels and high surface areas still remained available to contribute to the rapid and abundant adsorption of organic compounds. Elemental analysis data revealed that the carbon contents of P-MCM-41 and TFP-MCM-41 were $6.05 \%$ and $6.11 \%$, respectively, and that the number of $n$ propyl groups on P-MCM-41 was $1.68 \times 10^{-3} \mathrm{~mol} \mathrm{~g}^{-1}$ and the number of 3,3,3-trifluoropropyl groups on TFP-MCM-41 was $1.70 \times 10^{-3} \mathrm{~mol} \mathrm{~g}^{-1}$.

The hydrophilic-hydrophobic performance of material can be estimated by observing its behavior when in contact with water. As shown in Fig. 6, unmodified MCM-41 showed strong hydrophilicity as its water contact angle was $20.5^{\circ}$, and once put it into water, it sank to the bottom immediately. The contact angle of P-MCM-41 increased to $86.1^{\circ}$. When P-MCM-41 was put into water, it floated on the water surface first, but the edge of powder sample wetted gradually, so that some sample sank to the bottom or remained suspended in solution. However, the contact angle of TFP-MCM-41 was up to $151.0^{\circ}$, and it remained floating on the surface of water. The greater hydrophobicity of TFP-MCM-41 compared to that of P-MCM-41 suggests that the F atoms in the modifying agent played a major role in the hydrophobicity of the adsorbent.

\subsection{Kinetic study}

Adsorption is a time-dependent process, and it is important to know the rate of adsorption in order to better design and evaluate an adsorbent. Fig. 7 shows the time course of the DBP adsorption on MCM-41, P-MCM-41 and TFP-MCM-41 adsorbents. It can be seen that DBP adsorption onto the three adsorbents was fast in the first $60 \mathrm{~min}$, and then the rate of 
Table 1 Textural parameters of three unmodified and modified MCM-41 based adsorbents

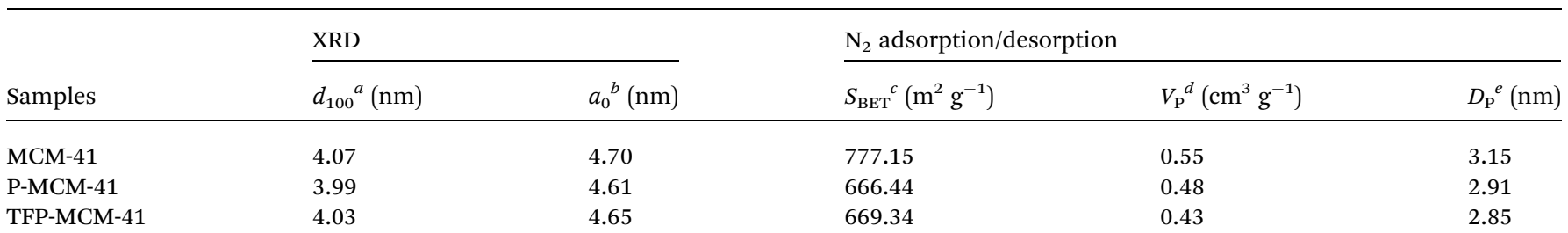

${ }^{a} d$-Value of (100) reflection. ${ }^{b} a_{0}=2 d_{100} / \sqrt{3}$ for hexagonal structures. ${ }^{c}$ BET surface area. ${ }^{d}$ Pore volume measured at $P / P_{0}=0.97 .{ }^{e}$ Average pore diameter calculated by BJH method.

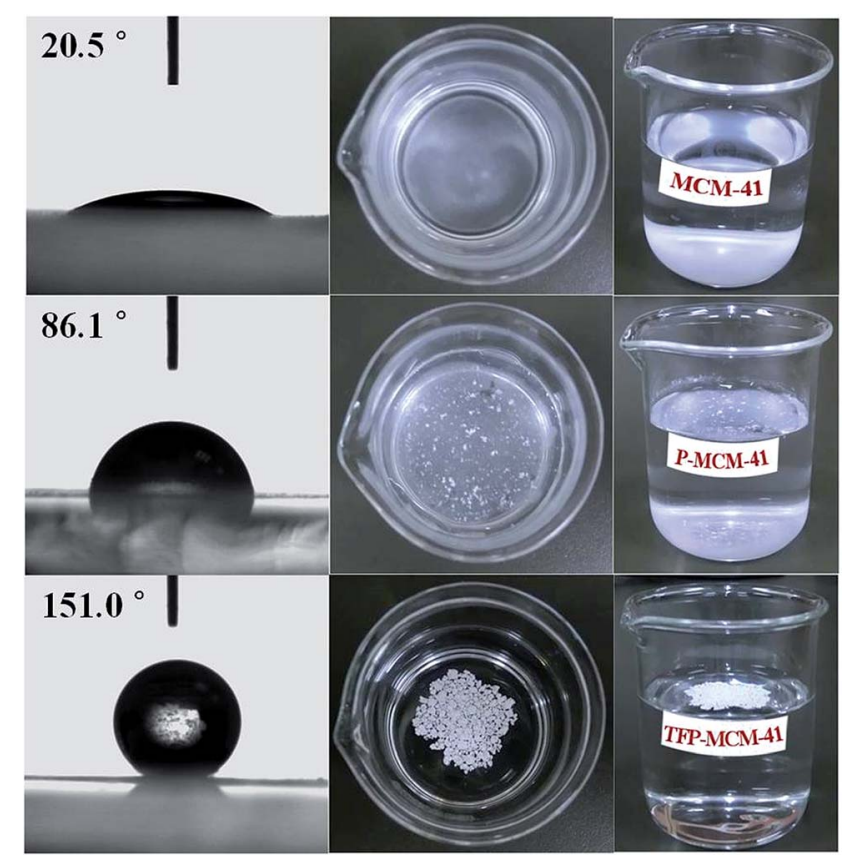

Fig. 6 Images of water contact angles of adsorbents (the left column) and graphs of apparent hydrophilic-hydrophobic property (two columns on the right).

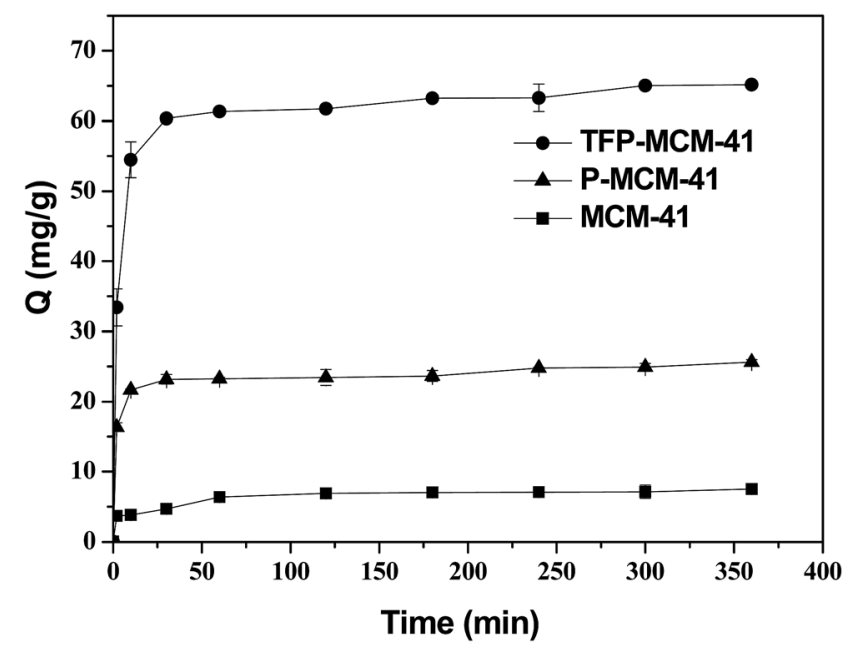

Fig. 7 The time course of adsorption of DBP on the three adsorbents (initial concentration: $5 \mathrm{mg} \mathrm{L}^{-1}$, adsorbent dose: $0.05 \mathrm{~g} \mathrm{~L}^{-1}$ ). adsorption of DBP slowed as the process neared equilibrium. This behavior can be attributed to the large number of vacant surface sites available for adsorption during the initial stage of the process. ${ }^{39}$

The amount of DBP adsorbed on the three adsorbents were in the following order: TFP-MCM-41 > P-MCM-41 > MCM-41. The DBP adsorption capacity of TFP-MCM-41 was $65.18 \mathrm{mg}$ $\mathrm{g}^{-1}$, which was about 8.7 times of that of MCM-41, and 2.5 times of that of P-MCM-41, respectively.

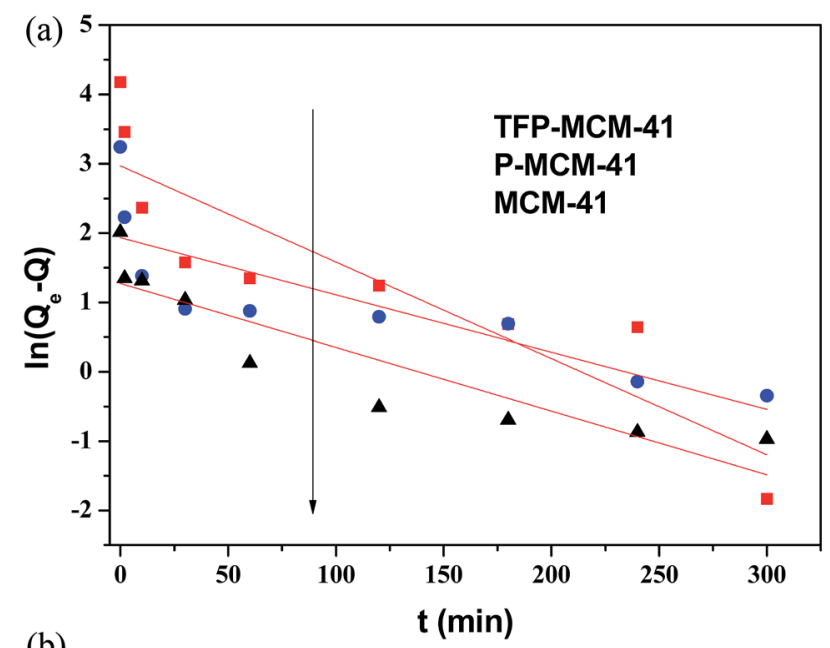

(b)

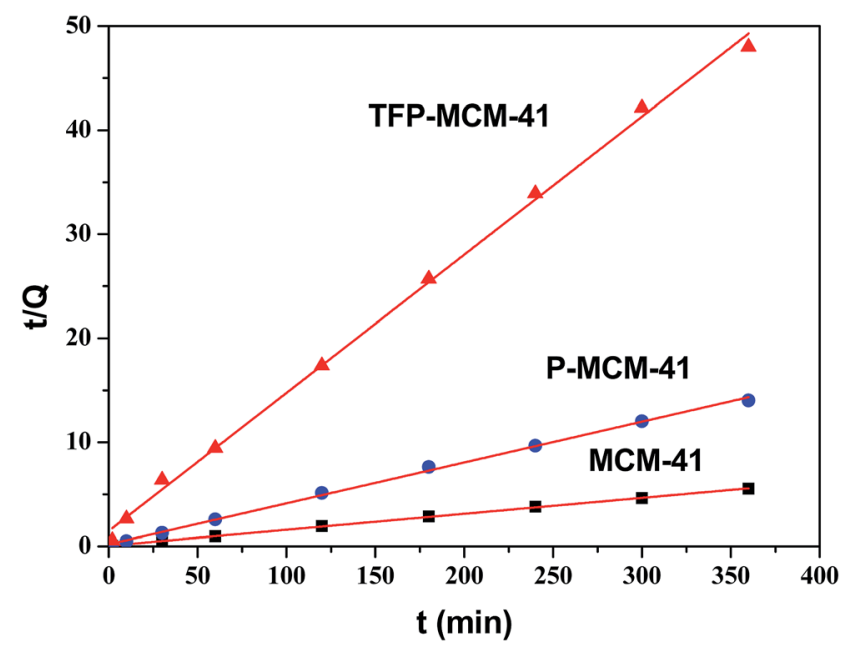

Fig. 8 Pseudo-first-order (a) and pseudo-second-order (b) kinetics plots for the adsorption of DBP on the three adsorbents. 
Table 2 Kinetic parameters for the adsorption of DBP on the three adsorbents

\begin{tabular}{|c|c|c|c|c|c|c|c|}
\hline Adsorbents & $Q_{e, \exp }\left(\mathrm{mg} \mathrm{g}^{-1}\right)$ & \multicolumn{3}{|c|}{ Pseudo-first order model } & \multicolumn{3}{|c|}{ Pseudo-second order model } \\
\hline P-MCM-41 & 25.65 & 6.92 & 0.0082 & 0.7955 & 25.44 & 0.007797 & 0.9986 \\
\hline TFP-MCM-41 & 65.18 & 19.53 & 0.0139 & 0.6883 & 65.36 & 0.004151 & 0.9996 \\
\hline
\end{tabular}

DBP is characteristic of strong hydrophobic organic compounds. The DBP adsorption capacity of MCM-41 is low because of the hydrophilic surface of the adsorbent. Due to the presence of $n$-propyl groups on the surface of P-MCM-41, the hydrophobic interactions between the $n$-propyl groups and the alkyl groups of DBP increased the DBP adsorption capacity of PMCM-41. For TFP-MCM-41 with 3,3,3-trifluoropropyl groups on surface, its hydrophobicity was higher than that of P-MCM-41. When TFP-MCM-41 was mixed with DBP, there were not only hydrophobic interactions between the 3,3,3-trifluoropropyl groups of adsorbents and alkyl groups of DBP, but also possible hydrogen bond interactions between the $\mathrm{H}$ atoms of DBP and the $\mathrm{F}$ atoms of the 3,3,3-trifluoropropyl groups. Because of the presence of these two interactions, TFP-MCM-41 presented much stronger affinity for DBP than did P-MCM-41.

The kinetics of adsorption describes the rate of adsorbate uptake on the adsorbent. The adsorption kinetic of DBP on all three adsorbents was investigated by fitting the experimental data with pseudo-first-order and pseudo-second-order models (as shown in Fig. 8). The slope and intercept of the plots were used to determine $k_{1}, k_{2}, R^{2}$, and $Q_{\mathrm{e} \text {,cal }}$. As reflected in the results in Table 2 and Fig. 8, the experimental kinetic data were better described as a pseudo-second order process than as a pseudofirst order process. This result is in agreement with the result of with Saeid Azizian ${ }^{40}$ who reported that the adsorption kinetics were better described by a pseudo-second-order model than a pseudo-first-order model when the initial concentration of solute $\left(C_{0}\right)$ was low.

\subsection{Adsorption isotherms}

The adsorption process can be further described by adsorption isotherms. The Langmuir, Freundlich and linear models are usually used to explore the adsorption isotherm in detail, being represented as:

Langmuir adsorption isotherm:

$$
Q_{\mathrm{e}}=Q_{\mathrm{m}} \frac{K_{\mathrm{L}} C_{\mathrm{e}}}{1+K_{\mathrm{L}} C_{\mathrm{e}}}
$$

where $Q_{\mathrm{e}}\left(\mathrm{mg} \mathrm{g}^{-1}\right)$ is the equilibrium adsorption capacity of DBP on the adsorbent; $C_{\mathrm{e}}\left(\mathrm{mg} \mathrm{L}^{-1}\right)$ is the concentration of DBP in equilibrium with the solution; $Q_{\mathrm{m}}\left(\mathrm{mg} \mathrm{g}^{-1}\right)$ is the maximum monolayer capacity of the adsorbent; and $K_{\mathrm{L}}$ is the Langmuir constant.

Freundlich adsorption isotherm:

$$
Q_{\mathrm{e}}=K_{\mathrm{F}} C_{\mathrm{e}^{\frac{1}{n}}}
$$

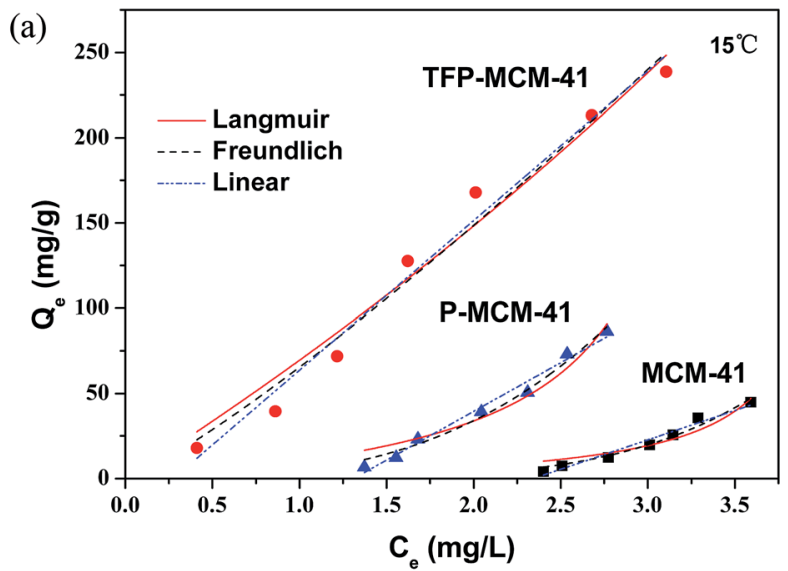

(b)

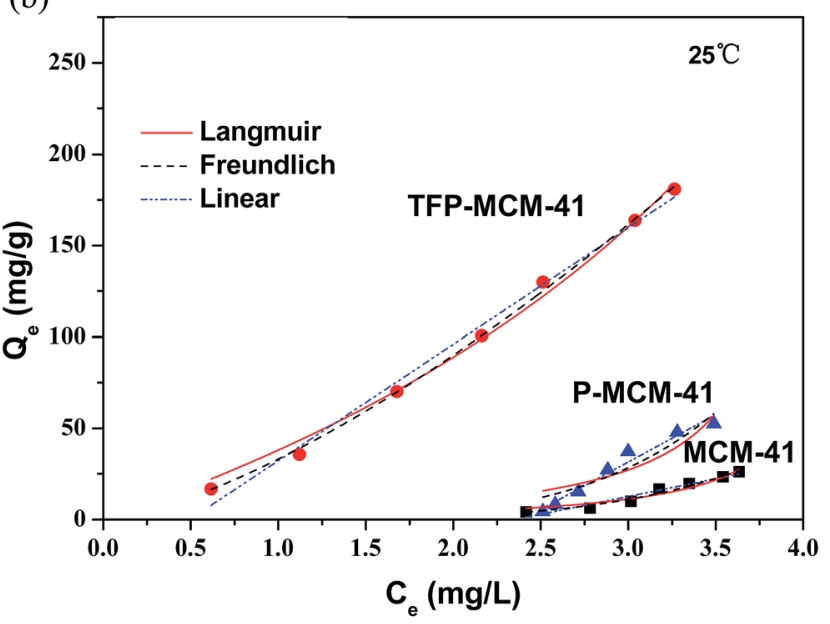

(c)

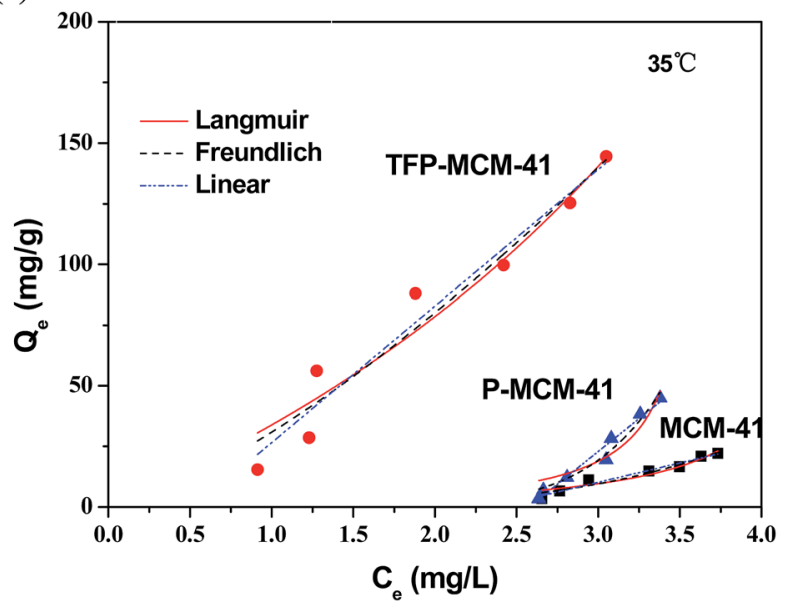

Fig. 9 Adsorption isotherms for DBP on the three adsorbents at different temperatures: (a) $15^{\circ} \mathrm{C}$, (b) $25^{\circ} \mathrm{C}$, (c) $35^{\circ} \mathrm{C}$. 
Table 3 Adsorption isotherms fitting parameters for DBP on the three adsorbents

\begin{tabular}{|c|c|c|c|c|c|c|c|c|c|}
\hline Adsorbent & $T\left({ }^{\circ} \mathrm{C}\right)$ & \multicolumn{3}{|l|}{ Langmuir } & \multicolumn{3}{|c|}{ Freundlich } & \multicolumn{2}{|l|}{ Linear } \\
\hline & 25 & 4.67 & 0.2350 & 0.9197 & 0.06585 & 4.66 & 0.9674 & 19.4626 & 0.9382 \\
\hline & 35 & 4.78 & 0.2219 & 0.9115 & 0.11974 & 3.98 & 0.9461 & 15.9561 & 0.9679 \\
\hline P-MCM-41 & 15 & 26.69 & 0.2795 & 0.9406 & 4.3379 & 2.97 & 0.9826 & 60.0644 & 0.9738 \\
\hline \multirow[t]{3}{*}{ TFP-MCM-41 } & 15 & 1122.00 & 0.05836 & 0.9659 & 65.4987 & 1.18 & 0.9733 & 87.7489 & 0.9804 \\
\hline & 25 & 263.71 & 0.1260 & 0.9916 & 33.1113 & 1.44 & 0.9981 & 63.7476 & 0.9907 \\
\hline & 35 & 242.99 & 0.1222 & 0.9296 & 30.7987 & 1.38 & 0.9392 & 56.3405 & 0.9512 \\
\hline
\end{tabular}

Table 4 Selected property values for DBP and Ph

\begin{tabular}{|c|c|c|c|c|c|}
\hline Compound & Abbreviation & Structural & $M_{\mathrm{w}}\left(\mathrm{g} \mathrm{mol}^{-1}\right)$ & $S_{\mathrm{w}}\left(25^{\circ} \mathrm{C}, \mathrm{mg} \mathrm{L}^{-1}\right)$ & $\log K_{\text {ow }}$ \\
\hline
\end{tabular}

where $K_{\mathrm{F}}$ and $1 / n$ are the Freundlich constants related to the adsorption capacity and adsorption intensity of the adsorbent, respectively. It is general in favor of adsorption when $1 / n$ is smaller, so the absorbability order of three adsorbents is TFPMCM-41 > P-MCM-41 > MCMC-41.

Linear adsorption isotherm:

$$
Q_{\mathrm{e}}=K_{\mathrm{d}} C_{\mathrm{e}}+b
$$

where $b$ and the distribution coefficient $K_{\mathrm{d}}$ are the constants.

Graphic presentations and the curve-fitting parameters of the adsorption isotherm results are given in Fig. 9 and Table 3. The amount of adsorbed DBP decreased with the increase in the temperature, indicating that the lower temperature was more favorable to adsorption. The adsorption isotherm of DBP on MCM-41 fit the Freundlich model better than the Langmuir model and liner model at all three temperatures $\left(R^{2}>0.94\right)$. However, the isotherm of DBP on P-MCM-41 and TFP-MCM-41 showed obvious linear trend, which was typical characteristic of hydrophobic adsorption. ${ }^{41}$ Besides, Fig. 9(a) showed that the DBP adsorption capacity of TFP-MCM-41 approached $250 \mathrm{mg}$ $\mathrm{g}^{-1}$ when the adsorption temperature was $15{ }^{\circ} \mathrm{C}$, which was much higher than that of P-MCM-41 $\left(83 \mathrm{mg} \mathrm{g}^{-1}\right)$ and unmodified MCM-41 (31 $\left.\mathrm{mg} \mathrm{g}^{-1}\right)$. The adsorption capacity of TFP-MCM41 for DBP also surpassed some other common adsorption materials, such as natural clay (about $0.7 \mathrm{mg} \mathrm{g}^{-1}$ ), ${ }^{42}$ biological adsorbent $\beta$-cyclodextrins $\left(1.7 \mathrm{mg} \mathrm{g}^{-1}\right){ }^{43}$ It also much higher than that of amphiphilic polypropylene nonwoven $(28.97 \mathrm{mg}$ $\left.\mathrm{g}^{-1}\right)^{44}$ and multilayer nanotubes with outer diameter of $10-$ $20 \mathrm{~nm}\left(99 \mathrm{mg} \mathrm{g}^{-1}\right) \cdot{ }^{45}$

\subsection{Selective adsorption performance of the adsorbents}

In order to investigate the adsorption selectivity of hydrophobic DBP, phenol $(\mathrm{Ph})$ was chosen as competitive adsorbant. $\mathrm{Ph}$ is relatively hydrophilic ( $\left.\log K_{\mathrm{ow}}=1.46\right)$ and is commonly found in many polluted waters. Selected properties of DBP and Ph are compared in Table 4 . The adsorption effect of TFP-MCM-41 and P-MCM-41 adsorbents in aqueous mixtures with different concentration ratios of DBP and $\mathrm{Ph}\left(C_{\mathrm{DBP}}: C_{\mathrm{Ph}}\right)$ is illustrated in Fig. 10. The selective adsorption coefficients of the two adsorbents for DBP $\left(K_{\mathrm{DBP}}\right)$ are listed in Table 5 . The amount of DBP adsorbed on TFP-MCM-41 was not affected by the concentration of $\mathrm{Ph}$ in the mixtures, and was significantly larger than the amount of $\mathrm{Ph}$ adsorbed. When the concentration of $\mathrm{Ph}$ was the same as DBP, the selective adsorption coefficient of TFP-MCM41 for DBP $\left(K_{\mathrm{DBP}}\right)$ was as high as 486.6 . Since TFP-MCM- 41 has a slight affinity for phenol, $K_{\mathrm{DBP}}$ increased generally with the increase in the concentration of phenol. These data show that in the mixtures of DBP and Ph, TFP-MCM-41 has remarkable adsorption selectivity for the highly hydrophobic DBP. In contrast, P-MCM-41 showed a lesser degree of selectivity for DBP due to adsorbent's lesser hydrophobicity. With the increase in the $\mathrm{Ph}$ concentration, the amount of $\mathrm{Ph}$ adsorbed on P-MCM-41 increased while the adsorption of DBP became weaker due to the competitive adsorption. In short, the greater the hydrophobicity of the adsorbent, the greater the adsorption selectivity for the hydrophobic organic compounds.

Fig. 11 summarizes the situation: hydrophobic interactions between the DBP alkyl groups and $n$-propyl groups of P-MCM-41 results in greater selectivity for adsorption of DBP than with 

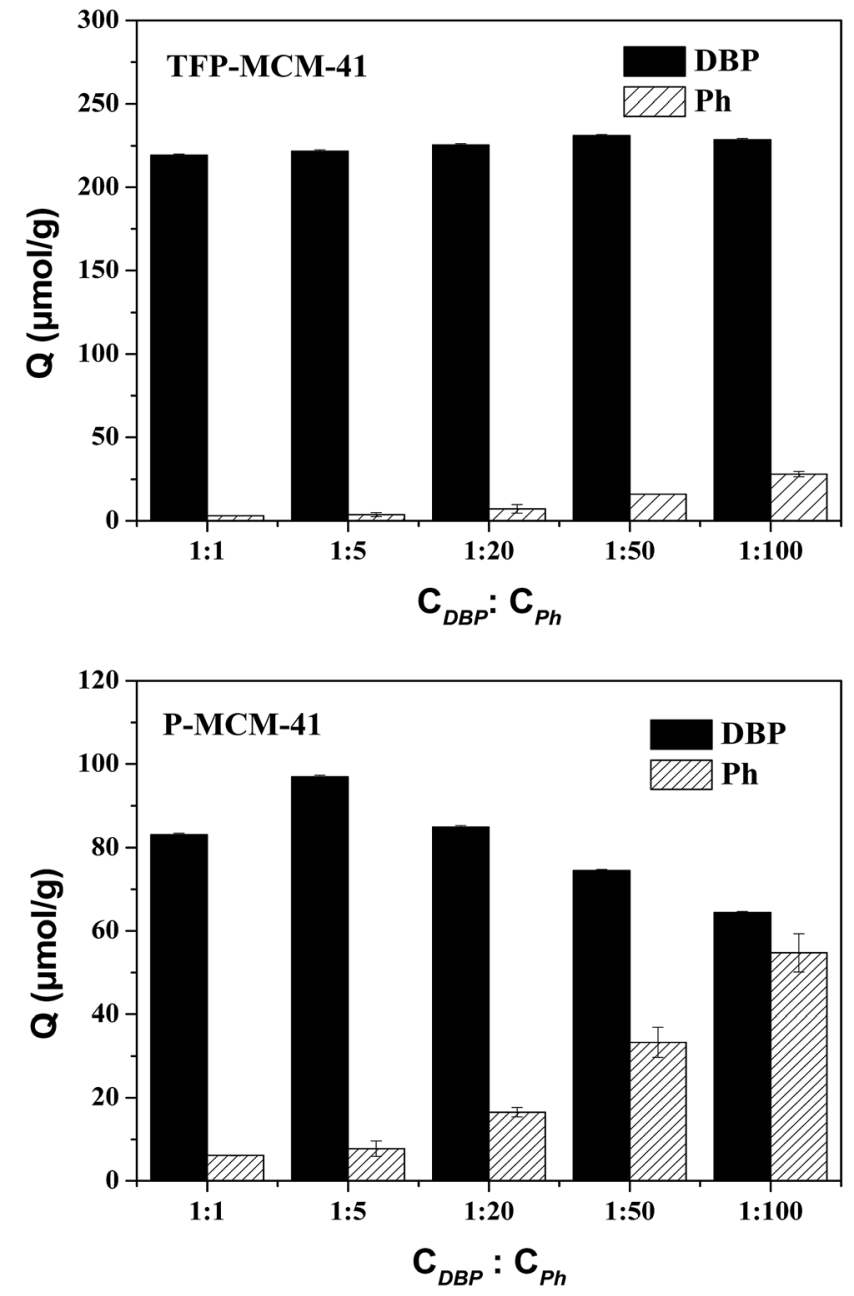

Fig. 10 The adsorption effect of two adsorbents in solutions with different $C_{\mathrm{DBP}}: C_{\mathrm{Ph}}$ values (initial concentration: $1.8 \times 10^{-5} \mathrm{~mol} \mathrm{~L}^{-1}$ (i.e. $5 \mathrm{mg} \mathrm{L}^{-1}$ ), adsorbent dose: $0.05 \mathrm{~g} \mathrm{~L}^{-1}$, contact time: $6 \mathrm{~h}$ ).

Table 5 Selective adsorption coefficient of DBP in mixed solutions with different $C_{\mathrm{DBP}}: C_{\mathrm{Ph}}$ ratios

\begin{tabular}{llrrrrr}
\hline$C_{\text {DBP }}: C_{\mathrm{Ph}}$ & $1: 1$ & $1: 5$ & $1: 20$ & $1: 50$ & $1: 100$ \\
\hline$K_{\mathrm{DBP}}$ & TFP-MCM-41 & 486.6 & 1718.8 & 3836.8 & 4417.4 & 4365.0 \\
& P-MCM-41 & 29.1 & 121.6 & 186.5 & 182.9 & 173.5
\end{tabular}

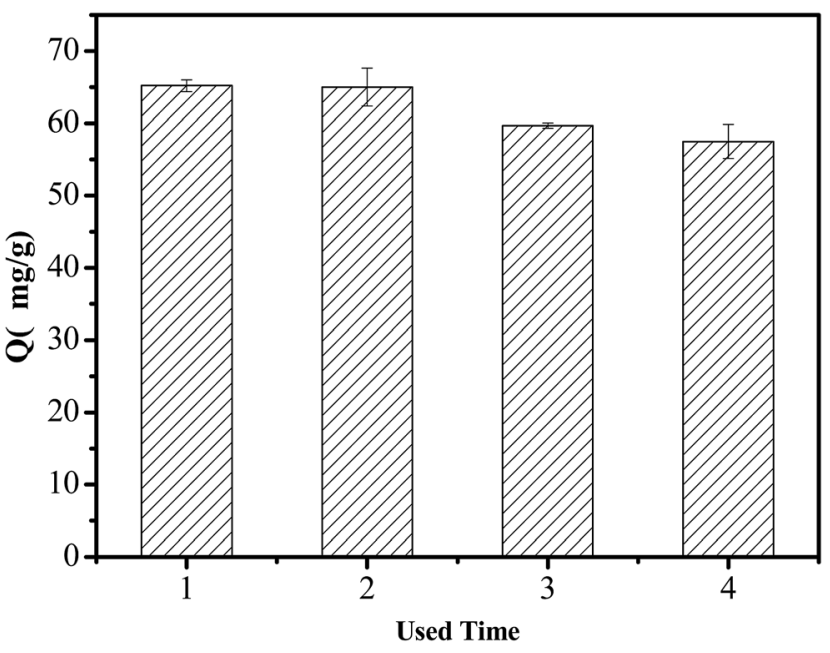

Fig. 12 The recyclability of TFP-MCM-41 for DBP removal.

MCM-41 alone. Even greater selectivity results from adsorption using TFP-MCM-41 due to similar hydrophobic interactions, plus hydrogen bond interactions between the $\mathrm{H}$ atoms of DBP and the $\mathrm{F}$ atoms of the 3,3,3-trifluoropropyl groups.

\subsection{Regeneration performance of the adsorbent}

The regeneration performance of the adsorbent is an important factor for the wide application, making it beneficial to reduce the cost of the adsorbent. $\mathrm{NaOH}$ solution $(0.1 \mathrm{M})$ was used to regenerate the saturated adsorbent. The regenerated adsorbent was reused in the next run under the same conditions. The regenerated and recycled results of TFP-MCM-41 are shown in Fig. 12. TFP-MCM-41 had a stable and excellent adsorption capacity for DBP after four cycles. The adsorption capacity of TFP-MCM-41 decreased slightly after three cycles, probably attributed to a partial loss of the organic functional groups from TFP-MCM-41 during the regeneration process. As a result, TFPMCM-41 could be a stable and excellent adsorbent for DBP removal.

\section{Conclusions}

In this work, we prepared a highly hydrophobic 3,3,3trifluoropropyl-functionalized mesoporous silica, TFP-MCM-41, by refluxing using 3,3,3-trifluoropropyltrimethoxysilane as a modifying agent. TFP-MCM-41 showed much stronger

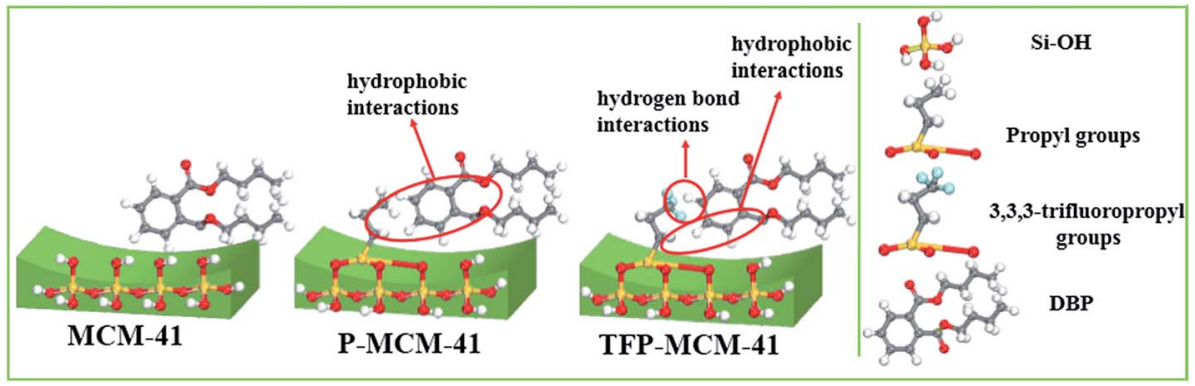

Fig. 11 Schematic illustration of the interactions between the three adsorbent and DBP. 
hydrophobicity and higher adsorption affinity for hydrophobic DBP than the $n$-propyl-functionalized P-MCM-41 or unmodified MCM-41. Especially, TFP-MCM-41 exhibited remarkable selectivity for DBP, which could preferentially take up DBP from the aqueous mixture containing small amounts of DBP and large quantities of phenol. The outstanding adsorption affinity and selectivity for DBP of TFP-MCM-41 can be ascribed to both hydrophobic interactions and hydrogen bond interactions that occur between the 3,3,3-trifluoropropyl groups and DBP molecules. This work provided a valuable guidance for developing the efficiently functionalized adsorbents for wastewater treatment.

\section{Acknowledgements}

This work was supported by National Natural Science Foundation of China (No. 21277051, 21577039), Science and Technology Planning Project of Guangdong Province, China (No. 2015A020215004), and the Fundamental Research Funds for the Central Universities, SCUT (No. 2015ZQ08, 2015KXKYJ07). We are grateful to Dr Donald G. Barnes for providing useful advice and language help to improve our paper.

\section{References}

1 F. Zeng, K. Cui, Z. Xie, L. Wu, D. Luo, L. Chen, Y. Lin, M. Liu and G. Sun, J. Hazard. Mater., 2009, 164, 1171.

2 N. H. Kleinsasser, B. C. Wallner, E. R. Kastenbauer, H. Weissacher and U. A. Harreus, Teratog., Carcinog., Mutagen., 2001, 21, 189.

3 F. Zeng, J. X. Wen, K. Y. Cui, L. N. Wu, M. Liu, Y. J. Li, Y. J. Lin, F. Zhu, Z. L. Ma and Z. X. Zeng, J. Hazard. Mater., 2009, 169, 719.

4 O. Bajt, G. Mailhot and M. Bolte, Appl. Catal., B, 2001, 33, 239.

5 E. Mylchreest, R. C. Cattley and P. Foster, Toxicol. Sci., 1998, 43, 47.

6 E. F. J. Group, Environ. Health Perspect., 1986, 65, 337.

7 Y. Liu, Z. L. Chen and J. M. Shen, J. Anal. Methods Chem., 2013, 2003, 419349.

8 A. Walcarius and L. Mercier, J. Mater. Chem., 2010, 20, 4478. 9 X. Zhang and L. Lei, J. Hazard. Mater., 2008, 153, 827-833.

10 Y. H. Wang, X. Q. Liu and G. Y. Meng, Mater. Res. Bull., 2008, 43, 1480-1491.

11 J. Marugán, J. Aguado, W. Gernjak and S. Malato, Catal. Today, 2007, 129, 59-68.

12 D. I. Petkowicz, R. Brambilla, C. Radtke, C. D. S. da Silva, Z. N. da Rocha, S. B. Pergher and J. H. dos Santos, Appl. Catal., A, 2009, 357, 125-134.

13 M. Mahalakshmi, S. V. Priya, B. Arabindoo, M. Palanichamy and V. Murugesan, J. Hazard. Mater., 2009, 161, 336-343.

14 M. Takeuchi, J. Deguchi, M. Hidaka, S. Sakai, K. Woo, P. P. Choi, J. K. Park and M. Anpo, Appl. Catal., B, 2009, 89, 406-410.

15 F. Quan, Y. Hu, X. Liu and C. Wei, Phys. Chem. Chem. Phys., 2015, 17, 19401.
16 B. Lu, Y. Ju, T. Abe and K. Kawamoto, RSC Adv., 2015, 5, 56444.

17 Y. Chen, W. Li, J. Wang, Q. Yang, Q. Hou and M. Ju, RSC Adv., 2016, 6, 70352.

18 T. Kasahara, K. Inumaru and S. Yamanaka, Microporous Mesoporous Mater., 2004, 76, 123.

19 L. Huang, H. Xiao and Y. Ni, Colloids Surf., A, 2004, 247, 129.

20 P. A. Mangrulkar, S. P. Kamble, J. Meshram and S. S. Rayalu, J. Hazard. Mater., 2008, 160, 414.

21 X. S. Zhao and G. Q. Lu, J. Phys. Chem. B, 1998, 102, 1556.

22 H. Huang, C. Yang, H. Zhang and M. Liu, Microporous Mesoporous Mater., 2008, 111, 254.

23 H. Yamashita, K. Maekawa, H. Nakao and M. Anpo, Appl. Surf. Sci., 2007, 237, 393.

24 Y. Kuwahara, K. Maki, Y. Matsumura, T. Kamegawa, K. Mori and H. Yamashita, J. Phys. Chem. C, 2009, 113, 1552.

25 H. Yamashita, S. Kawasaki, S. Yuan, K. Maekawa, M. Anpo and M. Matsumura, Catal. Today, 2007, 126, 375.

26 H. Yamashita, K. Maekawa, H. Nakao and M. Anpo, Appl. Surf. Sci., 2004, 237, 393.

27 L. C. Sang and M. O. Coppens, Phys. Chem. Chem. Phys., 2011, 13, 6689.

28 H. Y. Huang, C. L. Yang, H. X. Zhang and M. C. Liu, Microporous Mesoporous Mater., 2008, 111, 254.

29 A. Matsumoto, K. Tsutsumi, K. Schumacher and K. K. Unger, Langmuir, 2002, 18, 4014.

30 X. Y. Liu, L. B. Sun, F. Lu, T. T. Li and X. Q. Lu, J. Mater. Chem. A, 2015, 5, 1623.

31 H. Huang, C. Yang, H. Zhang and M. Liu, Microporous Mesoporous Mater., 2008, 111, 254.

32 X. S. Zhao and G. Q. Lu, J. Phys. Chem. B, 1998, 102, 1556.

33 S. Yuan, L. Shi, K. Mori and H. Yamashita, Microporous Mesoporous Mater., 2009, 117, 356.

34 Y. H. Kim, B. Lee, K. H. Choo and S. J. Choi, Microporous Mesoporous Mater., 2011, 138, 184.

35 L. C. Juang, C. C. Wang and C. K. Lee, Chemosphere, 2006, 64, 1920.

36 H. T. Zhao, K. L. Nagy, J. S. Waples and G. F. Vance, Environ. Sci. Technol., 2000, 34, 4822.

37 Y. Liu, Z. Gu, J. L. Margrave and V. N. Khabashesku, Chem. Mater., 2004, 20, 3924.

38 P. B. Sarawade, J. K. Kim, A. Hilonga and H. T. Kim, Powder Technol., 2010, 197, 288.

39 C. Pacurariu, G. Mihoc, A. Popa, S. Muntean and R. Ianos, Chem. Eng. J., 2013, 222, 218.

40 S. Azizian, J. Colloid Interface Sci., 2004, 276, 47.

41 T. E. M. Ten Hulscher and G. Cornelissen, Chemosphere, 1996, 32, 609.

42 H. Liu, D. Zhang, M. J. Li, L. Tong and L. Feng, Chemosphere, 2013, 92, 1542.

43 S. Murai, S. Imajo, Y. Takasu, K. Takahashi and K. Hattori, Environ. Sci. Technol., 1998, 32, 782.

44 X. Zhou, J. Wei, H. Zhang, K. Liu and H. Wang, J. Hazard. Mater., 2014, 273, 61.

45 F. Wang, J. Yao, K. Sun and B. S. Xing, Environ. Sci. Technol., 2010, 44, 6985. 